\title{
Transmission scheduling for wireless mesh networks with temporal reuse
}

\author{
Francois Pierre Sarel Luus ${ }^{*}$ and Bodhaswar Tikanath Jugpershad Maharaj
}

\begin{abstract}
Link-assigned transmission schedules with timeslot reuse by multiple links in both the space and time domains are investigated in this study for stationary multihop wireless mesh networks with both rate and power adaptivity. Specifically, cross-layer optimised schedules with proportionally fair end-to-end flow rates and network coding capability are constructed for networks operating under the physical interference model with single-path minimum hop routing. Extending transmission rights in a link-assigned schedule allows for network coding and temporal reuse, which increases timeslot usage efficiency when a scheduled link experiences packet depletion. The schedules that suffer from packet depletion are characterised, and a generic temporal reuse-aware achievable rate region is derived. Extensive computational experiments show improved schedule capacity, quality of service, power efficiency and benefit from network coding accrued with schedules optimised in the proposed temporal reuseaware convex rate region.
\end{abstract}

Keywords: Scheduling, temporal reuse, cross-layer optimisation, link-assignment, rate region

\section{Introduction}

Wireless mesh networking maintains a strong position in future communications, especially since it can extend access from a base station or gateway. This prominent implementation scenario can also provide flexible last mile access for stationary wireless mesh clients, instead of more expensive wired options. The focus of this paper is thus on the optimisation of transmission scheduling for stationary wireless mesh networks that use link-assigned scheduling with extended transmission rights. Although properly researched, the transmission scheduling aspect of wireless network design should be re-evaluated in the light of recent advances in network coding (NC) and cross-layer network optimisation. Specifically, modifications are required to allow linkassigned schedules to perform opportunistic network coding and to improve timeslot usage efficiency when the schedule is predefined.

Client meshing in a wireless mesh network creates data transport flows between all node pairs, which causes a traffic pattern where opposing flows traverse the network links. This presents a high likelihood for a

\footnotetext{
* Correspondence: fpsluus@gmail.com

Department of Electrical, Electronic and Computer Engineering, University of
} Pretoria, Pretoria 0002, South Africa

\section{Springer

(C) 2011 Luus and Maharaj; licensee Springer. This is an Open Access article distributed under the terms of the Creative Commons Attribution License (http://creativecommons.org/licenses/by/2.0), which permits unrestricted use, distribution, and reproduction in any medium, provided the original work is properly cited. combine packets intended for exchange between two neighbouring nodes into one transmission. Wireless mesh networks are replete with network coding opportunities which have the potential of significantly improving network throughput, quality of service and power efficiency. Opportunistic network coding is simple to deploy in an existing network when there is no opportunistic listening involved. Link-assigned scheduling is designed in this paper with support for network coding in mind, without incurring a lesser spatial reuse penalty or increased difficulty of cross-layer optimisation.

The two predominant transmission schedule assignment methods are node- and link-assignment, and while nodeassigned schedules $[1,2]$ allow for $\mathrm{NC}$ and have better usage efficiency, they lack the higher spatial reuse and finer granularity for optimisation achieved by link-assigned schedules [3-5]. Preference is given to link-assignment since it is more amenable to cross-layer optimisation, and it can achieve greater capacity owing to better spatial reuse. A novel hybrid node-link assignment method was presented in [6] (see also [7]), which keeps the high spatial reuse of link-assignment but integrates the NC capability and a constituent of the usage efficiency of node-assignment. This hybrid strategy was termed link-assignment with 
extended transmission rights (LET), where a node can attempt transmission on an alternative link if its primary scheduled link experiences packet depletion.

Packet depletion has been defined in [8] as the condition where a scheduled link does not have packets to transmit, resulting in a wasted timeslot with link-assigned scheduling. Conventional link-assignment only authorises one outgoing link per active node, and if that link is not utilised during its scheduled timeslot, then the opportunity and potential capacity are wasted. This lack of available packets for transmission on a link is a function of the network load, traffic pattern and transmission scheduling, and it is shown that this packet depletion occurs in most wireless mesh networks with client meshing where packets are added randomly to data flows. The hybrid assignment strategy provides alternative links for potential transmission, which can then make more efficient use of the available capacity in the case of primary link packet depletion. This beneficial aspect of some schedule assignment strategies in improving usage efficiency with multiple link options per node is analysed in this paper in terms of temporal reuse. The investigation of transmission scheduling with regard to temporal reuse is instructive in assisting with optimised schedule design, and it presents a novel viewpoint for the analysis of schedule assignment strategies.

The hybrid LET-schedules were cross-layer optimised in [8], according to the framework outlined in [9] (see also [10]). Specifically, cross-layer optimised schedules with proportionally fair end-to-end flow rates and network coding capability are constructed for networks operating under the physical interference model [11] with singlepath minimum hop routing. The adopted model does not capture the multi-carrier air interface, such as OFDM (orthogonal frequency-division multiplexing), but rather a single-channel physical interference model to place the focus on a capacity analysis for different transmission scheduling algorithms. The hybrid node-link assignment is redefined in terms of temporal reuse, which is proposed in this study as the time domain analogue of spatial reuse. The schedules that suffer from packet depletion and have the potential to benefit from temporal reuse are characterised, and an improved estimate of the achievable rate region is derived that incorporates the increased capacity afforded by temporal reuse.

The work in [8] is extended in this paper to include optimisation of networks with both variable transmission power and transmission at multiples of the base rate. When nodes can vary transmission power then the network spatial reuse potential and power efficiency can be increased, and when variable rates are allowed then network throughput and the benefit from network coding can be improved. Incorporating these changes into the temporal reuse-aware cross-layer optimisation is one of the unique contributions in this paper, where the capacity calculations, optimisation and complexity issues relating to variable power and rate control are dealt with.

Apart from the variable power and rate control, temporal reuse analysis and characterisation of networks that suffer from packet depletion, this paper further extends on the previous work of the authors in [8] by providing improved formulations for calculating the temporal reuse-aware rate region. The computationally feasible rate region is derived in a manner that enhances the understanding of the underlying factors that increase capacity with extended transmission rights. The capacity calculations and the optimisation program are also expanded to incorporate variable rate and power assignments, and the simulations include analyses of schedules with different rate and power objectives. In addition, a numerical analysis is done to determine the power efficiency of the various schedules considered in this paper. The main contribution is the calculation of the capacity region for wireless mesh networks that use predefined link-assigned transmission schedules with extended transmission rights and its use in a cross-layer optimisation framework similar to the one in [9].

Extensive computational experiments attest to the efficacy of the contributed generic scheduler in estimating the higher usage efficiency yielded by temporal reuse. Schedule capacity is measured as the constant number of packets per timeslot added to a random data flow such that a bounded mean end-to-end packet delay is conserved, and a greater capacity is achieved with the proposed temporal reuse-aware scheduler when compared to the non-aware scheduler for both schedules having temporal reuse. The capacity increases with $\mathrm{NC}$ are greater for the proposed scheduler, and in addition, flow rate fairness is improved for all combinations of rate/ power adaptivity and $\mathrm{NC}$ usage. Power efficiency, as related to the mean transmission power per packet, is also improved when the rate region used takes into account the benefits of temporal reuse.

The wireless networks and traffic models studied are defined in Section 2 and the achievable rate region and cross-layer optimisation framework for link-assigned schedules are reconsidered in Section 3. The role of temporal reuse in improving schedule performance is motivated in Section 4, where a new temporal reuse-aware rate region is also formulated. A generic temporal reuseaware schedule optimiser can be practically implemented with column generation according to the algorithm in Section 5, and the benefits of the new scheduler are determined in the computational experiments described in Section 6.

\section{System model}

Multihop wireless mesh networks with $N$ nodes $n \in \mathcal{N}$ and $L$ links $l \in \mathcal{L}$ are considered. Nodes are located at 
fixed positions, have isotropic antennas with mutually exclusive reception and transmission capabilities, and have infinitely large buffers. A directed wireless link $l$ (single-user Gaussian channel) is formed from a transmitting node $\operatorname{tr}(l)$ to a receiving node $\operatorname{re}(l)$ for a sufficiently high signal-to-interference and noise ratio (SINR)

$$
\frac{G_{\operatorname{tr}(l) \operatorname{re}(l)} P_{\operatorname{tr}(l)}}{\sigma_{\mathrm{re}(l)}+\sum_{n \neq \operatorname{tr}(l)} G_{n \mathrm{re}(l)} P_{n}}=\gamma_{l}(P) \geq \gamma_{\mathrm{tgt}}^{(1)}
$$

The transmit power of node $n$ is $P_{n}$ (limited to $0 \leq P_{n}$ $\left.\leq P_{\max }\right)$, the thermal noise at receiver $m$ is $\sigma_{m}$, and the effective power gain between transmitter $n$ and receiver $m$ is $G_{n m}$. The gain is calculated according to a deterministic fading model $G_{n m}=K_{n m} d_{n m}^{-\rho}$, where $K_{n m}$ is a normalisation constant, $d_{n m}$ is the Euclidean distance between $n$ and $m$, and $\rho$ is a constant path loss exponent. The link SINR $\gamma_{l}(P)$ is a function of the global power assignment (possible multiple transmitters owing to spatial reuse), which is denoted by $P=\left(P_{n}: n \in \mathcal{N}\right)$.

The target SINR for transmission at a discrete $r$-multiple of the base rate is $\gamma_{\mathrm{tgt}}^{(r)}$, where $r=1$ refers to the base transmission rate and $r=0$ a zero transmission rate. In practice, the link rate is limited to $c_{l}=c_{\mathrm{tgt}}^{(r)}=r W \log \left(1+\gamma_{\mathrm{tgt}}^{(1)}\right)$ when $\gamma_{\mathrm{tgt}}^{(r)} \leq \gamma_{l}(P)<\gamma_{\mathrm{tgt}}^{(r+1)}$, where $W$ is the system bandwidth in the Shannon capacity formulation. Links are activated during specific discrete timeslots, where all timeslot durations are equal and chosen such that one data packet can be forwarded in one timeslot. Perfect synchronisation between all nodes is assumed and every node operates according to a global transmission schedule.

The link gains are characterised in this paper only by the large-scale parameters, although realistically smallscale fading does exist even on the line-of-sight links between static nodes. CSI (channel state information) feedback is needed by the central scheduling agent in order to take the small-scale fading into account. However, since the focus is on centralised transmission scheduling, the main requirement is only that the network topology remains intact. By choosing a large SINR threshold (e.g. $10 \mathrm{~dB}$ ) for the links in the topology, the small-scale fading analysis becomes less important. This allows for the study to focus on the capacity analysis of a fixed topology and not on the dynamics of the possible changes in topology due to small-scale fading.

Data traffic is modelled as $F=N(N-1)$ transport flows $f \in \mathcal{F}$ between every possible source-sink node pair in the network, where data is generated at the source and delivered to the sink at flow rates $s=\left(s_{f}: f \in \mathcal{F}\right)$. Only single-path routing is considered in this study, and the link-flow incidence matrix $R \in$ $\mathbb{R}^{L \times F}$ couples the links with the flows so that

$$
R_{l f}=\left\{\begin{array}{l}
1 \text { if flow } f \text { uses link } l \\
0 \text { otherwise }
\end{array}\right.
$$

\section{Link-assigned scheduling}

The purpose of a transmission schedule is to control link transmission powers and rates, regulate medium access and provide adequate capacity for all network traffic. Transmission schedules are predetermined for a limited number of timeslots, with the frame being repeated in time. The capacity provided during a specific timeslot is defined by the associated link rate vector, which gives the transmission rate for each active link in the spatial reuse group, or clique. The cross-layer optimisation framework in [9] is partly reviewed in this section to make the paper self-contained.

\subsection{Link capacities}

Each link can be assigned a total of $r_{l, \max }+1$ different rates according to the link rate allocation policy $C_{l}=\left\{c_{\mathrm{tgt}}^{\left(r_{l}\right)}: r_{l} \in\left[0, r_{l, \max }\right]\right\}$, where $r_{l}=0$ means an inactive link and $r_{l}=r_{l, \max }$ the maximum possible multiple of the base rate for the link $l$. A link rate vector is one possible network capacity assignment for a single timeslot, so that every link in the network is assigned a specific rate in the link rate vector. All $K \ll \prod_{l \in \mathcal{L}}\left(r_{l, \max }+1\right)$ possible link rate vectors $\left\{v_{k}, k \in[1, K]\right\}$ are given by the set

$$
\mathcal{V}=\left\{v_{k}=\left(c_{l} \in C_{l}: l \in \mathcal{L}\right), \quad k \in[1, K]\right\}
$$

A schedule allocates a certain number of timeslots to each link rate vector, so that the long-term achievable link rate region can be defined as

$$
\mathcal{C}=\operatorname{conv}(\mathcal{V})=\left\{\begin{array}{l|l}
c=\sum_{\substack{k \in[1, K] \\
v_{k} \in \mathcal{V}}} \alpha_{k} v_{k} & \begin{array}{l}
\alpha_{k} \geq 0, \\
\sum_{k} \alpha_{k}=1, \\
k \in[1, K]
\end{array}
\end{array}\right\}
$$

This is similar to the capacity region for wireless ad hoc networks defined in [12]. The convex hull conv() of the set $\mathcal{V}$ includes all achievable average link capacities owing to time-sharing of all the link rate vectors. This convex combination of link rate vectors allocates a time fraction of $\alpha_{k}$ to every link vector $v_{k}$ to form the polyhedral rate region.

\subsection{Schedule optimisation}

Link capacities are represented by the transmission schedule, which can be optimised to maximise a global utility function. The level of satisfaction with a flow rate 
$s_{f}$ is given by a utility function $u\left(s_{f}\right)$, which is concave and strictly increasing. Maximising the weighted network throughput $\sum_{f \in \mathcal{F}} w_{f} s_{f}$ leads to grossly unfair flow rates [13], so a proportionally fair flow utility is decided on where $u \rightarrow-\infty$ when $s_{f} \rightarrow 0^{+}$. The flow rates $s$ are proportionally fair if and only if $u\left(s_{f}\right)=\log \left(s_{f}\right)$ (see [14]), and this leads to a higher total network throughput than with maximisation of a uniform flow rate.

Network throughput depends on the available link capacities, which are consumed with relaying potentially multiple flows. The aggregate traffic load on the links is given by the vector $R s$, which is due to the point-topoint data flows and the multihop nature of the mesh network. The achievable link rates are given by the capacity vector $c \in \mathcal{C}$, so that the flow rates are limited by the primary constraint $R s \leqslant c$, where $\leqslant$ denotes a component-wise inequality. The purpose of the optimisation problem is to find the time-sharing coefficients (let $\mathcal{K}=[1, K])($ let $\mathcal{K}=[1, K])$ for a capacity vector $c$ that maximises the global utility according to

$$
\begin{aligned}
& \text { maximise } \sum_{f} u\left(s_{f}\right) \\
& \text { subject to } R s \preccurlyeq c \\
& \qquad c \in \mathcal{C}, \quad s \succcurlyeq 0
\end{aligned}
$$

The concave global utility proposed in [9] is subject to convex constraints so it can be solved as a convex optimisation problem that produces a cross-layer optimised solution. The resultant schedule consists of link rate vectors $\left\{v_{k}: k \in \mathcal{K}\right\}$ and the associated time-sharing coefficients $\left\{\alpha_{k}: k \in \mathcal{K}\right\}$, which define transmission power and rate control in the physical layer and medium access control in the data link layer, respectively. The optimal solution involves at most $L+1$ link rate vectors according to Carathéodory's theorem [15] (see [16]), so there are at most $L+1$ non-zero $\alpha_{k}$ values in the optimal schedule. The exact number of utilised link rate vectors depends on the specific optimal solution for a particular network. The greater the number of links, the more numerous the possible spatial reuse groups, which increases the number of used link rate vectors. For a proper TDMA (time-division multiple access) schedule, it can be seen that $L$ link rate vectors would be needed for a feasible solution. When spatial reuse comes into play, less link rate vectors can describe the same capacity of TDMA, so the limit of the number of involved link rate vectors is not exceeded. The delivery route of each data flow is established in the network layer as represented by $R$, and the flow rates $s$ are regulated in the transport layer.

\section{Scheduling with temporal reuse}

Temporal reuse is important in transmission scheduling, as it is a means to share capacity and improve schedule usage efficiency. This term is explained further in this section, and the networks that can have improved efficiency with temporal reuse are also characterised. The capacity region for link-assigned scheduling with extended transmission rights is formulated, and a computationally feasible suboptimum is proposed. This accurate temporal reuse-aware capacity region is then used in the subsequent sections to obtain the full schedule optimisation program.

\subsection{Temporal reuse}

Spatial reuse is the reuse of a timeslot by simultaneously activated links, with sufficient SINR, in the spatial domain. Likewise, temporal reuse is defined here as the reuse of a timeslot by a group of links in the time domain. A temporal reuse group is the set of outgoing links from a particular node, which may transmit in a certain timeslot. Since a transmission schedule is composed of a $T$-length frame that is repeated in time, every timeslot in the frame is repeated at frequency $1 / T$. This means that the links in a temporal reuse group will share the capacity of a timeslot over all the repetitions of the timeslot in which the temporal reuse group appears. The reuse of a timeslot in the time domain thus refers to a timeslot, at a particular location in the scheduling frame, that is reused by a group of links as the specific timeslot reoccurs with the repetition of the frame in time.

The purpose of temporal reuse is to increase schedule capacity usage efficiency in the case of link-assigned schedules, but it is also important in node-assignment to allow all links to transmit even though only nodes are scheduled. Node-assigned schedules [1] have to employ a form of temporal reuse to provide capacity for every outgoing link associated with a scheduled node. Having multiple link options per node results in higher capacity usage efficiency, at the expense of reduced spatial reuse. Alternatively, link-assigned schedules with extended transmission rights can achieve some measure of the same improved efficiency with better spatial reuse, which results in a higher schedule capacity.

In the case of node-assigned scheduling, each node forms its own temporal reuse group and secondary scheduling must be executed at each node to service each of the outgoing links of a node. However, temporal reuse groups are prioritised in link-assigned scheduling with extended transmission rights, which makes the schedule completely defined since the secondary scheduling is implied. In addition, by extending a link assignment to a prioritised group of links, the schedule allows for the implementation of opportunistic network coding, which further improves performance, as in the case of nodeassigned scheduling. 


\subsection{Schedule utilisation efficiency}

A link must have a packet to transmit every timeslot that it is scheduled; otherwise, a usage inefficiency occurs due to packet depletion. The networks that can experience packet depletion and the associate usage inefficiencies are characterised in this subsection. Predefined schedule frames with a limited number of timeslots provide capacity $\pi_{l}$ for each link $l$, where $\pi_{l}$ packets per timeslot can be added to a data flow randomly chosen according to a uniform distribution, such that the schedule will be able to handle the resultant load on $l$.

Let $p_{l}$ be the probability that a packet added to a random flow will have to be routed on a link $l$, and let $T_{l} \approx c_{l}^{-1}$ be the average number of timeslots between the start of successive scheduled timeslots for link $l$ with continual repetitions of the scheduling frame. The link scheduling period $T_{l}$ is approximately the inverse of the link rate $c_{l}$, if the schedule is composed in a cyclical manner so that multiple schedulings of a link rate vector are as far removed in the scheduling frame as possible. Then, the average number of packets that have to be sent in a scheduled timeslot of link $l$ is given by $\eta_{l}(\pi)$ in (6) for a global flow load $\pi$, which also represents the efficiency of the schedule in terms of link $l$, where at most one packet can be transmitted per timeslot.

$$
\eta_{l}(\pi)=\sum_{i=1}^{\left\lfloor\pi T_{l}\right\rfloor} i \prod_{j=0}^{i-1} \frac{\left(\left\lfloor\pi T_{l}\right\rfloor-j\right)}{j+1}\left(p_{l}\right)^{i}\left(1-p_{l}\right)^{\left\lfloor\pi T_{l}-i\right\rfloor}
$$

This formulation sums the products of the probability that a certain number of $i$ packets will be added to $l$ in between successive schedulings of $l$ with the number of packets $i$, to get the average number of added packets for $l$ in each of its scheduled timeslots. This is a monotonically increasing function that relates a global load to a locally resultant load at a specific link $l$. The full efficiency load for link $l$ is then the solution to $\eta_{l}\left(\pi_{l}\right)=1$, which equates to the applied global load that will fully utilise the specific link capacity provided by the schedule. The globally applied flow load is upper bounded by the link capacity that is saturated first; thus, the maximum packet load per timeslot is $\widehat{\pi}=\min _{l \in \mathcal{L}} \pi$. Thus, for every link with $\pi_{m}>\widehat{\pi}$, the average utilisation efficiency is $\eta_{m}(\widehat{\pi})<1$, which means that the maximum load allowed is too small to make full use of the available capacity for link $m$.

Full schedule utilisation can thus only be achieved when $\eta_{l}^{-1}(1)=\eta_{m}^{-1}(1), \forall l, m \in \mathcal{L}$; otherwise, for any inequality $\pi_{l}<\pi_{m}, l \neq m$, it must mean that $\eta_{l}\left(\pi_{l}\right)<1$. The global load may not exceed the minimum $\pi_{l}$, because then link $l$ will experience a load greater than its capacity, so only when the full efficiency loads for all the links are equal can every link be saturated without exceeding the capacity of any one link. For networks with spatial reuse and multihop flows, it becomes more difficult to calculate schedules that give equal full efficiency load $\pi_{l}$ for each link $l \in \mathcal{L}$, and even if it is achieved, there is still no trivial guarantee that the schedule will achieve optimal schedule capacity and fairness. The optimisation problem is thus further constrained by $\eta_{l}^{-1}(1)=\eta_{m}^{-1}(1), \forall l, m \in \mathcal{L}$, which mainly constrains the link scheduling periods $T_{l}$, rather than flow-link probabilities $p_{l}$ which are determined by the network topology and routing.

By extending transmission rights to alternative links in the case of packet depletion, the unused timeslots of the unsaturated links can be used to increase the capacity for most of the links in the network to $\pi_{m}+\varepsilon_{m}$. Consequently, the maximum schedule capacity will be increased to $\widehat{\pi}=\min _{l \in \mathcal{L}}\left(\pi_{l}+\varepsilon_{l}\right)$, and the schedule will have a higher possible usage efficiency due to the applied temporal reuse.

\subsection{Link capacities with temporal reuse}

Given a scheduled link rate vector $v_{k} \in \mathcal{V}$ that experiences packet depletion for one of the associated links $\mathcal{L}\left(v_{k}\right)=\left\{l \in \mathcal{L}: v_{k}(l)>0\right\}$, the scheduler can resort to transmission on an alternative link through temporal reuse. In such a timeslot where an alternate link is used, another link rate vector $v_{m} \neq v_{k}$ describes the capacity used for the temporal reuse instance. All possible link rate vectors that can describe temporal reuse for the primary vector $v_{k}$ are defined by the extended set $\mathcal{V}\left(v_{k}\right)=\left\{v_{m} \in \mathcal{V}: P\left(v_{k}\right) \succcurlyeq P\left(v_{m}\right)\right\}$.

An extended set of link rate vectors can be associated with any one specific link rate vector, such that spatial reuse is maintained but with increased temporal reuse. The node transmission powers for a link rate vector $v_{k}$ are given by $P\left(v_{k}\right)$, and the power assignment should not be exceeded by any alternate link rate vector.

\subsubsection{Temporal reuse-aware rate region}

The average link capacities used for a scheduled extended set depend on its ordering policy, the network topology, radio propagation model and traffic load, as these factors influence the prevalence of packet depletion. For all repetitions of a timeslot where an extended set of $v_{k}$ is scheduled, a certain fraction $\phi_{k m}$ of repetitions will be represented by every link rate vector $v_{m} \in \mathcal{V}\left(v_{k}\right)$ in the extended set. These fractions can only be determined by simulating the network, although the values $\Phi(\mathcal{V})=\left\{\left\{\varphi_{k m}: \sum \varphi_{k m} \leq 1, v_{m} \in \mathcal{V}\left(v_{k}\right)\right\}: v_{k} \in \mathcal{V}\right\} \quad$ will change when a schedule is used that is optimised using those values in the temporal reuse-aware rate region

$$
\mathcal{C}_{e}=\left\{\begin{array}{l|l}
\sum_{\substack{k \in \mathcal{K} \\
v_{k} \in \mathcal{V}}} \alpha_{k} \sum_{v_{m} \in \mathcal{V}\left(v_{k}\right)} \varphi_{k m} v_{m} & \begin{array}{l}
\alpha_{k} \geq 0, \\
\sum_{k} \alpha_{k}=1, \\
k \in \mathcal{K}
\end{array}
\end{array}\right\}
$$


The aware rate region $C_{e}$ gives the achievable link capacities with the extended temporal reuse sets when taking the inefficiencies due to packet depletion into account. Optimising a schedule with more accurate representations of the link rate vectors leads to improved network performance.

\subsubsection{Rate region estimation}

A schedule optimisation algorithm should be independent of a specific network topology and radio propagation model, so several assumptions and simplifications are applied to create a generic optimiser.

(i) Each alternative link in the temporal reuse set must operate at the maximum possible transmission rate, within the power constraints.

(ii) The usage probabilities of links in a temporal reuse set are determined only on the basis of the order in which the links are queried for packets in the case of packet depletion, regardless of network topology or radio propagation model. The validity of this assumption is tested in the usage efficiency analysis in the simulation section, where it is shown that it is reasonable.

Since some links in a temporal reuse set could have sufficient SINR to transmit at different rates, only the highest rate (primary criterion) is chosen to maximise the capacity of each extended set. For schedules that use link rate vectors with variable power control, a minimum power criterion (secondary with a small $\varepsilon>0$ ) could also be used to differentiate between multiple link rate vectors with a maximum rate for a specific link. The temporal reuse set for a clique node $n \in \mathcal{T}\left(v_{k}\right)=\left\{n \in \mathcal{N}: \mathcal{L}\left(v_{k}\right) \cap \mathcal{O}(n) \neq \emptyset\right\}$, where $\mathcal{O}(n)$ are the outgoing links of node $n$, which is thus calculated as $\mathcal{L}_{n}\left(v_{k}\right)=\left\{l \in \mathcal{L}: c_{l}>0, c_{l} \in C_{n}\left(v_{k}\right)\right\}$ where

$$
C_{n}\left(v_{k}\right)=\left\{\arg _{v_{m}(l)} \max _{v_{m} \in \mathcal{V}\left(v_{k}\right)}\left(v_{m}(l)-\varepsilon P\left(v_{m}, n\right)\right): l \in \mathcal{O}(n)\right\}
$$

To calculate the rate region $\mathcal{C}_{e}$, a very large set of $|\Phi(\mathcal{V})|=\sum_{v_{k} \in \mathcal{V}}\left|\mathcal{V}\left(v_{k}\right)\right|$ values particular to $\mathcal{V}$ is needed. A network-agnostic rate region can be rendered by focusing on the usage probabilities of alternative links in $\mathcal{L}_{n}\left(v_{k}\right)$, instead of alternative link rate vectors as in the case of $\Phi(\mathcal{V})$. With temporal reuse, when a primary scheduled link experiences packet depletion, the alternative links are queried in a certain order for available packets, according to the ordering policy, until a usable link is found.

Each link $l$ in a temporal reuse set $\mathcal{L}_{n}\left(v_{k}\right)$ is assigned an ordering value $\Theta_{n}\left(v_{k}, l\right) \in\left[0,\left|\mathcal{L}_{n}\left(v_{k}\right)\right|-1\right]$, where a smaller value means a higher priority, to establish an ordering policy, so a link $l \in \mathcal{L}_{n}\left(v_{k}\right)$ with a higher priority $\Theta_{n}\left(v_{k}, l\right)<\Theta_{n}\left(v_{k}, m\right)$ than $m \in \mathcal{L}_{n}\left(v_{k}\right)$ will have a higher usage probability $\mathcal{U}_{n}\left(v_{k}, l\right)>\mathcal{U}_{n}\left(v_{k}, m\right)$. The number of usage probabilities is reduced with the assumption that the utilisation probabilities are approximately equal when the usage priorities are the same, regardless of differences in networks, the extended sets, the particular node temporal reuse sets or links in question. This simplification is made on the basis that it is the order in which links in the temporal reuse set are checked that determines the link usage probabilities. Let $U$ be the simplified priority-based link usage probabilities, where the usage probability for a link with priority $\theta$ is $U_{\theta}$.

$$
U=\left\{U_{\theta} \in[0,1]: \sum_{\theta} U_{\theta} \leq 1, U_{\theta-i}>U_{\theta}, 0 \leq i \leq \theta, \forall \theta \geq 0\right\}
$$

The primary outgoing link for a node, i.e. the active link that is originally implicated in a $v_{k}$, has priority $\theta=$ 0 and lower priority reserve links have higher $\theta$ values. The simplified usage probabilities of outgoing links in the temporal reuse set for a specific node $n$ active in $v_{k}$ are given by

$$
\mathcal{U}_{n}\left(v_{k}\right)=\left\{\beta_{l}: l \in \mathcal{O}(n)\right\} \subseteq U
$$

Although this form does not define a specific ordering policy, one based on relative traffic flow magnitudes is presented in the following subsection. Let $I_{L}$ be the identity matrix in $\mathbb{R}^{L \times L}$, where $I_{L}(l)$ is the $j$ th column in $I_{L}$, so that $j$ is the index of link $l$ in $\mathcal{L}$. Temporal reuse link sets are mutually exclusive; thus, for any $v_{k} \in \mathcal{V}$, the relationship $\cap_{n \in \mathcal{T}\left(v_{k}\right)} \mathcal{L}_{n}\left(v_{k}\right)=\emptyset$ holds, so the usage probability vector for a $v_{k}$ with temporal reuse is given by

$$
\mathcal{U}\left(v_{k}\right)=\sum_{n \in \mathcal{T}\left(v_{k}\right)} \sum_{l \in \mathcal{O}(n)} \mathcal{U}_{n}\left(v_{k}, l\right) I_{L}(l)
$$

The full link rate template for $v_{k}$ with temporal reuse given by $C\left(v_{k}\right)$ represents the non-achievable capacity before taking the actual usage limits specified by $\mathcal{U}\left(v_{k}\right)$ into account.

$$
C\left(v_{k}\right)=\sum_{n \in \mathcal{T}\left(v_{k}\right)} \sum_{l \in \mathcal{O}(n)} C_{n}\left(v_{k}, l\right) I_{L}(l)
$$

The Hadamard (Schur) product of the usage probability estimates $\mathcal{U}\left(v_{k}\right)$ and the rate template $C\left(v_{k}\right)$ give the resultant achievable capacity for $v_{k}$ with full temporal reuse, so the simplification of $\mathcal{C}_{e}$ gives the estimated achievable rate region for networks with spatial and temporal reuse.

$$
\widetilde{\mathcal{C}}_{e}=\left\{\begin{array}{l|l}
\sum_{\substack{k \in \mathcal{K} \\
v_{k} \in \mathcal{V}}} \alpha_{k}\left(\mathcal{U}\left(v_{k}\right) \odot C\left(v_{k}\right)\right) & \begin{array}{l}
\alpha_{k} \geq 0, \\
\sum_{k} \alpha_{k}=1, \\
k \in \mathcal{K}
\end{array}
\end{array}\right\}
$$


Apart from the time-sharing coefficients $\left\{\alpha_{k}: k \in \mathcal{K}\right\}$ and the link rate vector power assignments $\left\{P\left(v_{k}\right): v_{k} \in \mathcal{V}\right\}$, each schedule has to include the prioritized temporal reuse link rate sets $\left\{\Theta\left(v_{k}\right), \mathcal{U}\left(v_{k}\right), C\left(v_{k}\right): v_{k} \in \mathcal{V}\right\}$ associated with every active transmitter node $n$ in the spatial reuse node set (clique) $\mathcal{T}\left(v_{k}\right)$ for every $v_{k} \in \mathcal{V}$. The schedule is composed by cycling between the maximum of $L+1$ involved link rate vectors until there are $\left\lceil\alpha_{k} \delta(L+1)\right\rceil$ occurrences of each vector $v_{k}$. A value of $\delta=3$ has been found to give good results for various network sizes.

$U$ is independent of a particular network topology or radio propagation model and can be determined once with the averaged values of simulations of many different networks and used to optimise any network afterwards, as done in the numerical analysis of Section 6 . The number of utilisation probability values needed to calculate $\widetilde{\mathcal{C}_{e}}$ is

$$
|U|=\max _{\substack{n \in \mathcal{T}\left(v_{k}\right) \\ v_{k} \in \mathcal{V}}}\left|\mathcal{L}_{n}\left(v_{k}\right)\right| \ll|\Phi(\mathcal{V})|
$$

This estimate $\widetilde{\mathcal{C}_{e}}$ is thus greatly simplified while maintaining the effect of temporal reuse on the usage efficiency and achievable capacity of link-assigned schedules.

\subsubsection{Ordering policy}

The discriminator that is used to assign priorities to the alternative links in a temporal reuse set is taken as the number of data flows that traverse a link. The more flows that have to be routed by a link, the greater the need for capacity; thus, preference is given to links that relay more flows. This traffic flow-based ordering policy assigns higher priority to the higher traffic link as formulated by

$$
\begin{aligned}
\operatorname{sgn} & \left(\mathcal{U}_{n}\left(v_{k}, l\right)-\mathcal{U}_{n}\left(v_{k}, m\right)\right) \\
= & \begin{cases}+1 \text { when } \sum_{f} R_{l f}>\sum_{f} R_{m f}, \\
0 \text { never, } \\
-1 \text { when } \sum_{f} R_{l f} \leq \sum_{f} R_{m f}\end{cases} \\
& \text { for } l \neq m, \forall l, m \in \mathcal{O}(n), \forall n \in \mathcal{T}\left(v_{k}\right)
\end{aligned}
$$

\section{Column generation approach}

The global utility maximisation problem in (5) determines the time-sharing coefficients for each link rate vector in $\mathcal{V}$. The number of link rate vectors is exponential in the number of network links, so calculating and storing them in order to solve (5) becomes a hurdle as the network grows. The solution also tends to be more inaccurate with computer solvers when more link rate vectors are involved.

For these reasons, the mathematical programming technique of column generation [17] is employed, where modifications specific to temporal reuse are made to the algorithm discussed in [9], which is reconsidered in this section. Optimisation can then be started with only a small number of link rate vectors, and more vectors that contribute most to increasing the global utility are added through column generation. The process of optimising a schedule with column generation, for networks that employ both spatial and temporal reuse, is explained in this section.

\subsection{Main algorithm}

The utility maximisation problem guides the column generation by providing information pertaining to capacity demands, so it is referred to as the master problem. The full master problem in (5) contains all $\mathcal{K}$ link rate vectors, but it is restricted to a subset $\mathcal{J} \subseteq \mathcal{K}$ of vectors in the restricted master problem (16) used for column generation.

$$
\begin{aligned}
\operatorname{maximize} & \sum_{f} u\left(s_{f}\right) \\
\text { subject to } & R s \preccurlyeq c, \quad s \succcurlyeq 0 \\
& c=\sum_{\substack{k \in \mathcal{J} \subseteq \mathcal{K} \\
v_{k} \in \mathcal{V}}} \alpha_{k}\left(\mathcal{U}\left(v_{k}\right) \odot C\left(v_{k}\right)\right) \\
& \sum_{k} \alpha_{k}=1, \quad \alpha_{k} \geq 0, k \in \mathcal{J} \subseteq \mathcal{K}
\end{aligned}
$$

When solving the restricted master problem, the capacity demand $\lambda_{l}$ for each link $l$ can be determined, which indicates the relative utility improvement for a unit increase in link capacity. For $u(\cdot)=\log (\cdot)$, each link demand also equals the inverse flow rate of the flow that only uses the corresponding link [18]. These capacity demands are given by the Lagrangian duality for (16), which is as follows.

$$
L(s, c, \lambda)=\sum_{f} u\left(s_{f}\right)-\lambda^{T}(c-R s)
$$

The optimal Lagrangian multipliers $\lambda^{*}$ are used to update $\mathcal{J}$ by maximising the upper bound on the optimum (weak duality, see [9]) according to

$$
u_{u}=\sup _{s \succcurlyeq 0}\left\{\sum_{f} u\left(s_{f}\right)-\lambda^{* T} R s\right\}+\sup _{c \in \widetilde{\mathcal{C}}_{e}}\left\{\lambda^{* T} c\right\}
$$

The lower bound (19) involves only the columns generated thus far, as indicated by $\mathcal{J}$, to find the best supply vector.

$$
u_{l}=\sup _{s \succcurlyeq 0}\left\{\sum_{f} u\left(s_{f}\right)-\lambda^{* T} R s\right\}+\sup _{c \in \widetilde{\mathcal{C}}_{e}^{\mathcal{J}}}\left\{\lambda^{* T} c\right\}
$$

Finding a new link rate vector from the $\mathcal{K}$-element rate region $\widetilde{\mathcal{C}_{e}}$ that maximises capacity for the given demand solves the scheduling subproblem $\sup _{c \in \widetilde{\mathcal{C}}_{e}}\left\{\lambda^{* T} c\right\}$. An extreme point in $\widetilde{\mathcal{C}}_{e}$ will maximise capacity [9] rather than a combination of link rate vectors. This generated 
column does not require that all $\mathcal{K}$ elements have to be known, but the new vector can be obtained by solving a mixed integer linear programming formulation discussed in the proceeding subsection.

Optimality is reached when the new link rate vector cannot supply a greater capacity than an existing vector. The process of solving the restricted master problem and expanding $\mathcal{J}$ is continued until the difference between the optimal upper bound $u_{u}$ and lower bound $u_{l}$ becomes smaller than a certain threshold $\varepsilon$, as shown by (20).

$$
u_{u}-u_{l}=\sup _{c \in \widetilde{\mathcal{C}}_{e}}\left\{\lambda^{* T} c\right\}-\sup _{c \in \widetilde{\mathcal{C}}_{e}^{J}}\left\{\lambda^{* T} c\right\}<\varepsilon
$$

The main column generation algorithm that is used to optimise a schedule, by forming $\mathcal{J}$ to maximise the global utility, is as follows:

1) Initialise the index set $\mathcal{J}$ with the link rate vectors that correspond to a simple TDMA link-assigned schedule operating at base rates only with no spatial reuse.

2) Solve the restricted master problem to optimality with a primal-dual interior-point method [19] to obtain the optimal Lagrangian multipliers or capacity demands $\lambda^{*}$.

3) Add the new link rate vector, that solves the scheduling subproblem, to $\mathcal{J}$.

4) Repeat the process until $u_{u}-u_{l}<\varepsilon$, or when the generated column is already present in $\mathcal{J}$.

\subsection{Scheduling subproblem}

The optimal Lagrangian multipliers $\lambda_{l}^{*}$ give the sensitivity of the lower optimum bound $u_{l}$ at the optimal point $c^{*}$. By satisfying the links with the greatest demand for capacity as indicated by $\lambda_{l}^{*}$, the lower bound can be improved most. By finding an extreme vertice in $c \in \widetilde{\mathcal{C}_{e}}$ to maximize $\lambda_{l}^{* T} c$, a new scheduling element is added to increase the network utility. This is termed the scheduling subproblem, and for networks with temporal reuse, it is formulated as finding

$$
\max _{v_{k} \in \mathcal{V}}\left(\lambda_{l}^{* T} \sum_{n \in \mathcal{T}\left(v_{k}\right)} \sum_{l \in \mathcal{O}(n)} \mathcal{U}_{n}\left(v_{k}, l\right) C_{n}\left(v_{k}, l\right) I_{L}(l)\right)
$$

A mixed integer linear program is used to determine which spatial reuse clique with extended transmission rights solves the scheduling subproblem. Let the binary variable $x_{l}^{(r)}$ indicate whether link $l$ is operating at $r$ times the base rate.

$$
x_{l}^{(r)} \in\{0,1\}, \quad \forall l \in \mathcal{L}, \forall r
$$

The binary variable $y_{n}$ specifies whether node $n$ is transmitting or not, so the following definition is included:

$$
y_{n} \in\{0,1\}, \quad \forall n \in \mathcal{N}
$$

Whenever a link is transmitting at a non-zero rate, the associated node also has to be activated, so this relationship is formulated as

$$
\sum_{r} x_{l}^{(r)}=y_{\operatorname{tr}(l)}, \quad \forall l \in \mathcal{L}
$$

In the program, the instantaneous maximum transmission power of a node $n$ is given by $P_{n}$ which is limited to a maximum as shown in (25):

$$
0 \leq P_{n} \leq P_{\max }, \forall n \in \mathcal{N}
$$

To ensure that all transmitting links have a sufficiently large SINR, the following linear constraint is imposed:

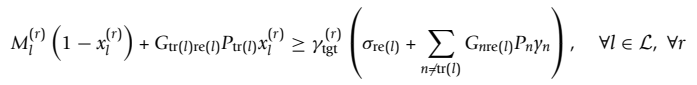

By choosing a sufficiently large $M_{l}^{(r)}$, the above constraint will be met when the link is not transmitting, so the following value will suffice:

$$
M_{l}^{(r)}=G_{\mathrm{tr}(l) \mathrm{re}(l)} P_{\mathrm{tr}(l)}+\gamma_{\mathrm{tgt}}^{(r)}\left(\sigma_{\mathrm{re}(l)}+\sum_{n \neq \mathrm{tr}(l)} G_{n \mathrm{nre}(l)} P_{n}\right), \quad \forall l \in \mathcal{L}, \forall r
$$

For scheduling with fixed power, the node transmit powers are fixed at $P_{n}=P_{\max }, \forall n \in \mathcal{N}$, and for nonadaptive rate scheduling the rate is limited to $r \in\{0,1\}$. Given the optimal Lagrange multipliers, the variable rate and power mixed integer linear program, which considers all possible temporal reuse priority policies, is thus formulated as

$$
\begin{aligned}
& \text { maximise } \sum_{n \in \mathcal{N}} \sum_{l \in \mathcal{O}(n)} \lambda_{l} \beta_{l} \sum_{r} c_{\mathrm{tg}}^{(r)} x_{l}^{(r)} \\
& \text { subject to }\left\{\beta_{l}: l \in \mathcal{O}(n)\right\} \subseteq U, \quad \forall n \in \mathcal{N} \\
& \\
&(22),(23),(24),(25),(26),(27)
\end{aligned}
$$

By ensuring that the links with greater traffic flow loads are assigned higher rates in a temporal reuse link set, the complexity can be reduced. The complexity of the scheduling subproblem still remains NP-hard, however. The following constraint can be added to the linear program to achieve the complexity reduction:

$$
\begin{aligned}
& \operatorname{sgn}\left(\sum_{r=a}^{r_{\max }} x_{l}^{(r)}-\sum_{r=a}^{r_{\max }} x_{m}^{(r)}\right) \\
& =\left\{\begin{array}{l}
+1 \text { when } \sum_{f} R_{l f}>\sum_{f} R_{m f}, \\
0 \text { when } \sum_{f} R_{l f}=\sum_{f} R_{m f}, \\
-1 \text { when } \sum_{f} R_{l f}<\sum_{f} R_{m f}
\end{array}\right. \\
& \text { for } l \neq m, \forall l, m \in \mathcal{L}, a \in\left[1, r_{\max }\right]
\end{aligned}
$$




\section{Numerical analysis}

Extensive simulation was conducted to evaluate the performance of $\widetilde{\mathcal{C}}_{e}$-schedules optimised in the temporal reuseaware rate region as compared to $\mathcal{C}$-schedules optimised in the default non-aware rate region. The simulation model is expounded on, and results are included that show the improvements that are gained with the addition of temporal reuse. The usage probability vector $U$ is estimated through simulation, and the process is explained and values given for networks with differing rate/power adaptivity. Schedule performance is gauged according to schedule capacity, fairness and power efficiency, and in this section, the benefits of the temporal reuse-aware rate region are made clear. Finally, the relationship between spatial and temporal reuse for the various schedules is investigated.

\subsection{Simulation model}

A radio link model is used where nodes transmit omnidirectionally at a maximum possible instantaneous power of $0.1 \mathrm{~W}$ and experience background receiver noise power of $\sigma=3.34 \times 10^{-12} \mathrm{~W}$, where an absolute circuitry temperature of $290^{\circ} \mathrm{K}$ and a noise figure of 10 are assumed. The deterministic fading model $G_{n m}=10^{-4} d_{n m}^{-3}$ is used with a path loss exponent of $\rho=3$, transmitter and receiver gains of 1 and a $1 \mathrm{~m}$ reference path loss ratio of $K_{n m}=10^{-4}$. The threshold SINR for base rate transmission is set at $\gamma_{\mathrm{tgt}}^{(1)}=10 \mathrm{~dB}$ and a shared bandwidth of $W=83.5 \mathrm{MHz}$ is used. This model corresponds broadly to that of an indoor wireless LAN using the entire 2.4-2.4835 GHz ISM band.

Multihop wireless mesh networks of 10 to 30 nodes are randomly generated in two dimensions where the maximum length of each dimension for 10, 15, 20, 25 and 30 node networks are set at 140, 160, 180, 200 and $220 \mathrm{~m}$ respectively. The various square dimensions have been experimentally chosen to produce fully connected networks with a good average spatial reuse potential that increases as the number of nodes increases. Singlepath minimum hop routing is used according to Dijkstra's algorithm, and only networks with a path between every node pair are considered. For each data point in the results, the averaged outcomes of 120 random networks are taken and each network size is analysed separately.

Schedules are determined centrally and shared with all nodes, so that with perfect synchronisation each node operates according to the schedule. Each data flow is communicated with packets where each packet can be relayed over one hop in one timeslot when transmitting at the base rate. The schedule capacity is determined as the maximum number of packets $\widehat{\pi}$ that can be added per timeslot to a flow randomly chosen according to a uniform distribution, such that the mean end-to-end packet delays are bounded. The numerical analysis determines the mean end-to-end packet delays as well as the mean flow rates and their standard deviation and the mean transmission power per packet at the maximum schedule capacity.

The analyses with $\mathrm{NC}$ use opportunistic two-way network coding [20] where a relay node XORs two packets intended for exchange between two neighbouring nodes. Neighbouring nodes do not need to communicate which packets each received, neither is opportunistic listening required with two-way coding, and the throughput gain with $n$-way coding is maximised when $n=2$. First-infirst-out (FIFO) transmit buffers are employed and with $\mathrm{NC}$ the buffer is checked in a FIFO manner to obtain the first two packets that can be coded and transmitted over the scheduled temporal reuse set.

\subsection{Improvements with temporal reuse}

The effect of temporal reuse on schedule capacity is determined for schedules optimised in the unaware rate region $\mathcal{C}$ with transmission rights extended afterwards. This $\mathcal{C}$-optimisation does not consider the capacity changes that extended transmission rights cause and is thus temporal reuse-unaware. Every link rate vector $v_{k}$ that is activated in a $\mathcal{C}$-schedule has transmission rights extended to $\left|\mathcal{L}_{n}\left(v_{k}\right)\right|-1=q-1$ alternative links for $q>0$ to see what improvements in the schedule capacity results.

As the number of alternative links is increased, the schedule capacity $\widehat{\pi}$ also increases, as shown in Figure 1a. So as transmission rights are extended, the added capacity $\min _{l \in \mathcal{L}} \varepsilon_{l}(q+n)>\min _{l \in \mathcal{L}} \varepsilon_{l}(q)$ expands for $n>0$, where $\varepsilon_{l}$ is a function of $q$, with greater improvements seen for smaller network sizes owing to smaller spatial reuse and thus better temporal reuse. The data population sizes for the large-extreme end of the $x$-axis are much smaller, since not all networks can achieve such large temporal reuse. This data population size inequality and the trade-off between the schedule capacity and endto-end packet delays cause the reverse trend in the packet delay values at a large temporal reuse.

The schedule optimised in the convex rate region $\mathcal{C}$ is named CVXOPT-LET in Figure 1, where LET refers to link-assignment with extended transmission rights (full temporal reuse). The mean end-to-end packet delays for the schedule operating at capacity are given in Figure 1b and are reduced for most of the rights extension. Thus, both the schedule capacity and the average end-to-end packet delays can be improved with temporal reuse.

\subsection{Estimation of usage efficiency}

Temporal reuse-unaware $\mathcal{C}$-optimised schedules with full rights extension (full temporal reuse) are analysed to estimate the usage probabilities $U$ of links in temporal reuse sets based on their assigned priorities $\theta$. The average 


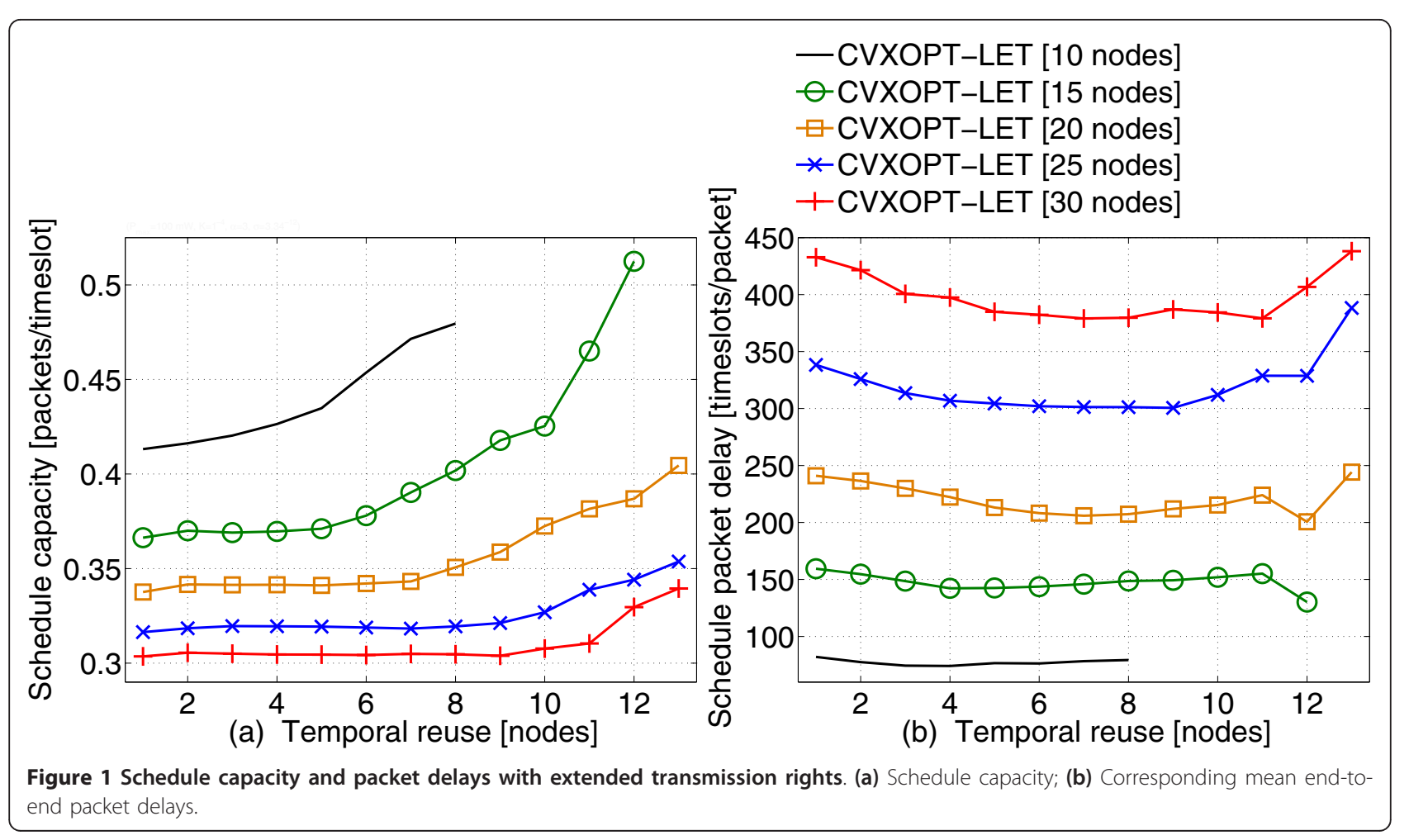

usage probabilities of 10-30 node networks are given in Table 1 for networks operating with schedules that have no power-rate adaptivity, power adaptivity only, rate adaptivity only and both power and rate adaptivity.

The mean (for different power-rate adaptivities) of the standard deviations due to networks having different sizes is given in the column for $S D A$. The accuracy of the given usage probabilities was determined by comparing them to that of temporal reuse-aware $\widetilde{\mathcal{C}}_{e}$-schedules and is given in the Acc. column. The validity of the assumption that different network realisations of the same size have similar usage probabilities is measured with the mean (for different power-rate adaptivities) of the mean (for different network sizes) of the standard deviations due to different network topologies as given in the column for $S D B$. The deviations are small

Table 1 Prioritised usage probabilities for power-rate adaptive networks

\begin{tabular}{cccccccc}
\hline $\boldsymbol{\theta}$ & CVX & Power & Rate & Power rate & SD A & SD B & Acc. \\
\hline 0 & 0.706 & 0.804 & 0.687 & 0.791 & 0.016 & 0.056 & 0.94 \\
1 & 0.170 & 0.125 & 0.179 & 0.130 & 0.005 & 0.022 & 0.79 \\
2 & 0.057 & 0.039 & 0.061 & 0.042 & 0.003 & 0.014 & 0.88 \\
3 & 0.029 & 0.017 & 0.031 & 0.019 & 0.003 & 0.010 & 0.88 \\
4 & 0.017 & 0.008 & 0.019 & 0.009 & 0.003 & 0.008 & 0.85 \\
5 & 0.010 & 0.004 & 0.011 & 0.005 & 0.002 & 0.006 & 0.81 \\
6 & 0.006 & 0.002 & 0.006 & 0.002 & 0.001 & 0.004 & 0.77 \\
\hline
\end{tabular}

compared to the means for the higher priority links, so the assumption is taken as sound.

Each column displays the effect of temporal reuse, which shares capacity among the temporal reuse set to increase timeslot usage efficiency. The primary links (corresponding to $\theta=0$ ) are not fully used, which indicates significant packet depletion occurring owing to unequal link capacities in proportionally fair cross-layer optimised schedules. These results thus show the important benefits that can be derived from temporal reuse.

\subsection{Convergence of the master problem}

The number of spatial reuse groups is limited for sizeconstrained fixed-topology networks, and the resulting link rate vectors are all determined. The accurate temporal reuse-aware link rate vectors are also determinable, and the number of vectors remains the same. The column generation must therefore end, since the number of link rate vectors is limited. Due to Carathéodory's theorem, there is a high probability that the master problem will converge before all the link rate vectors have been generated. The moment no new link rate vector can increase the capacity supply, the column generation converges.

Slater's condition is met for fully connected networks, since there is a strictly feasible solution for the master problem. One such solution is given by a simple linkassigned TDMA schedule that supplies a non-zero 
positive capacity $\tilde{c}_{l}$ for each link $l$ in the network. The network is fully connected so a non-zero positive flow rate $\tilde{s}_{p}$ can be achieved by every flow $p$ in the network, and if a flow rate of $\min _{l}\left\{\tilde{c}_{l} / \sum_{p} r_{l p}\right\}$ is assigned to each flow, then the convex constraint $R \tilde{s} \preccurlyeq \tilde{c}$ met. Since Slater's condition is met strong duality holds, and there is thus no duality gap. This means that the column generation must converge.

The convergence of the column generation for the temporal reuse-aware $\widetilde{\mathcal{C}_{e}}$-schedule with extended transmission rights is depicted in Figure 2. The reduction in the difference between the lower and upper bounds of the master problem is shown for different network sizes. The mean convergence rate was measured for 120 random network realisations, for each network size. Since larger networks have more spatial reuse groups and data flows, the convergence takes more iterations and the starting difference between the bounds is greater. The absence of the duality gap is confirmed, since all of the solutions converge with zero difference between the lower and upper bounds.

\subsection{Schedule performance comparison}

The practical superiority of schedule optimisation in the temporal reuse-aware rate region is proven through measurements of schedule capacity, flow rate fairness and transmit power efficiency.

\subsubsection{Schedule capacity}

Schedule capacity is taken as the primary schedule performance measure, and it is the maximum number of packets per timeslot that can be added to a random flow, so that the schedule maintains a limited mean end-to-end packet delay. The $\mathcal{C}$-schedule (CVX) capacity is increased with extended transmission rights (CVX-
LET) and when network coding is used (CVX-LET-NC), the capacity is increased even further, as shown in Figure 3a. The temporal reuse-aware $\widetilde{\mathcal{C}_{e}}$-schedule with extended transmission rights (CVXAware) has greater capacity than with CVX-LET, and the capacity improvement with NC (CVXAware-NC) is also notably larger than with CVX-LET-NC. The temporal reuse-aware rate region gives greater capacity to link rate vectors with good temporal reuse, which provides more $\mathrm{NC}$ opportunities for the $\widetilde{\mathcal{C}}_{e}$-schedule.

Reference graphs include those of TDMA, STDMA, traffic-adaptive STDMA with temporal reuse and NC (STDMA-Adaptive-LET-NC), and traffic-adaptive nodeassigned schedules with NC (Node-Assigned-NC). The STDMA schedule uses a packing heuristic [21], which packs as many links as have not yet transmitted into a timeslot, and the traffic-adaptive STDMA schedule is generated according to [22]. The node-assigned schedule lets a node choose a link with probability proportional to the number of flows that traverse the link, to achieve traffic adaptivity.

The mean end-to-end packet delays for the different schedules operating at their respective maximum capacities are given in Figure 3b. Node-assigned schedules have the lowest delay, although they do not have the capacity of the cross-layer optimised link-assigned schedules. The $\mathcal{C}$-schedule (CVX) achieves lower delays when temporal reuse (CVX-LET) is enabled, even though the delays for CVX are taken at a smaller load. The timeslot duration is a function of the packet length, which affects the quality of service if the packet delays grow large. Although apart from the proportional fairness, the end-to-end packet delays are allowed to grow as large as possible while remaining bounded. This is warranted to obtain the

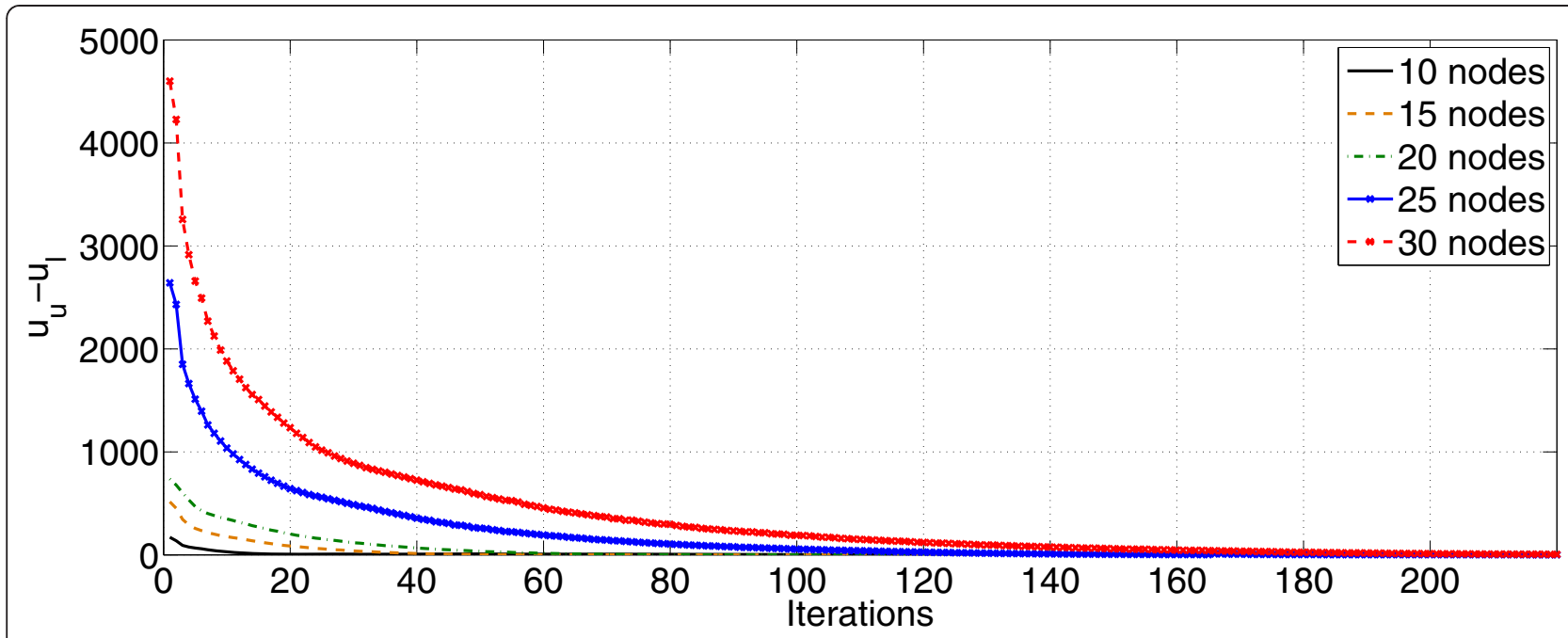

Figure 2 Convergence of the column generation approach to schedule optimisation. 


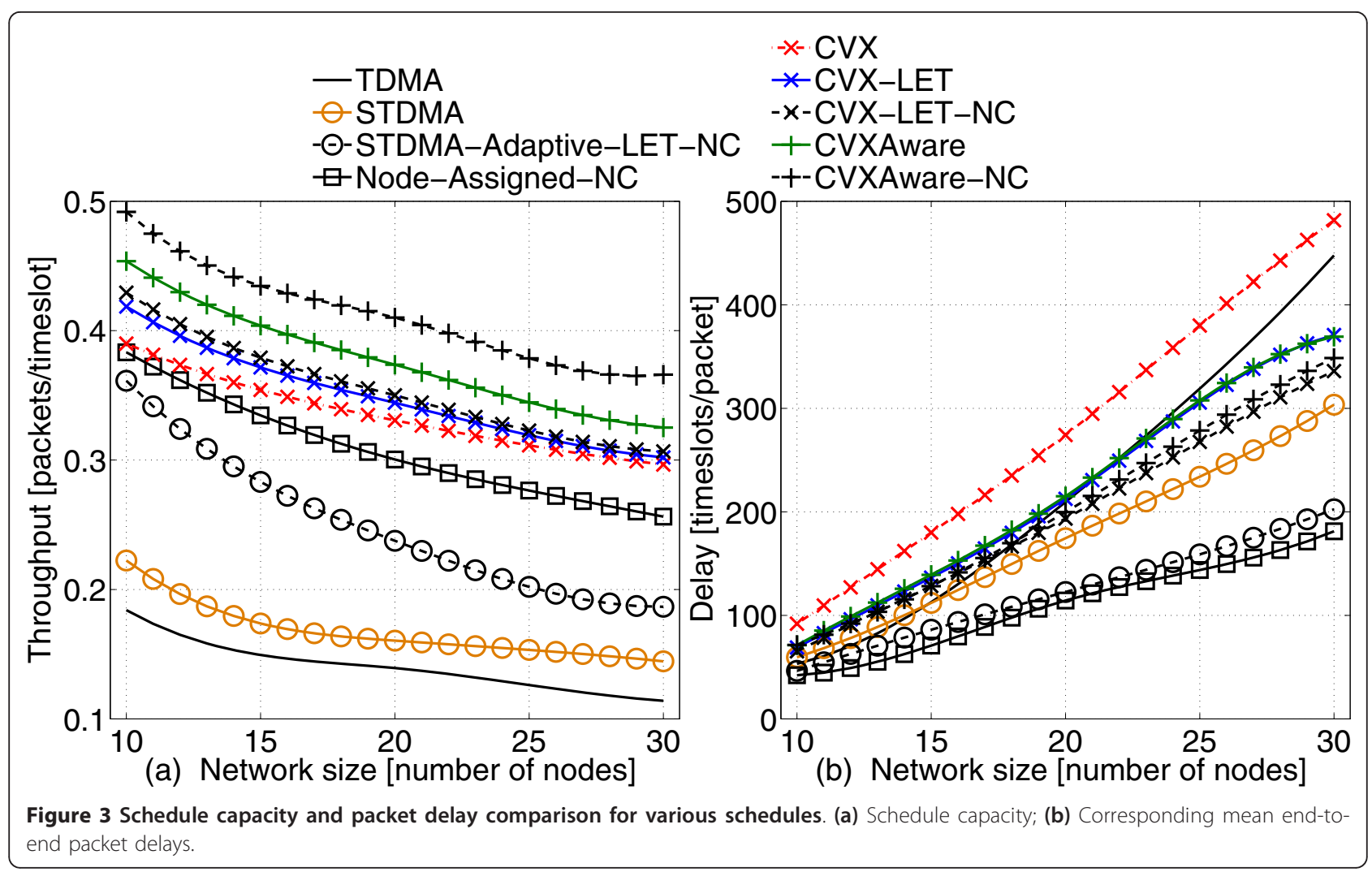

maximum schedule capacity, which is a more important metric in this study than a secondary quality of service concern.

The effect of rate and power adaptivity on the capacity of the cross-layer optimised schedules is shown in Figure 4. Schedules with power adaptivity (CVX-PowerLET, CVX-RatePower-LET, CVXAware-Power, CVXAware-RatePower) perform better because of more diverse spatial reuse considerations during optimisation. When $\mathrm{NC}$ is enabled for the temporal reuse-aware schedules (CVXAware), greater capacity increases are seen than with the $\mathcal{C}$-schedules, as shown in Figure 4b.

\subsubsection{Flow rate fairness}

The second important schedule performance measure is flow rate fairness, which indicates the quality of service level that can be expected with a schedule. For the schedules operating at maximum capacity, the associated mean flow rates are compared in Figure 5a, where nodeassigned schedules have the highest rate and CVX the lowest. Node-assigned schedules achieve the highest mean flow rate at the expense of rate fairness, and the best fairness is achieved by CVX as measured by the flow rate standard deviations in Figure 5b. The sum of the logarithms of the achieved flow rates during network simulation can also be used to assess proportional fairness, but the standard deviation of the achieved flow rates is calculated instead to accompany the mean achieved flow rate. The temporal reuse-aware schedule (CVXAware) achieves a better fairness than the unaware (CVX-LET) schedule, and fairness is not compromised with the use of $\mathrm{NC}$ for the CVXAware schedule.

For the rate-power adaptive schedules, significantly lower flow rate deviation occurs with the temporal reuseaware schedules than with the unaware schedules, since the proportionally fair global utility is more accurately determined when taking the benefits of temporal reuse into account. The improved fairness with the $\widetilde{\mathcal{C}}_{e}$-schedules is especially apparent with the power-adaptive schedules as shown in Figure 6b.

A network routes most of its data flows through the centre of the network, so by giving a centralised link more capacity, there can be more flows enjoying a higher transmission rate. This may increase the mean flow rate at the expense of reduced fairness, due to the reduced capacity experienced by flows routed across links on the edges of the network. A proportional fairness utility can balance the schedule capacity and fairness objectives explicitly, as opposed to the disregard of fairness by schedules such as node-assignment.

\subsubsection{Transmit power efficiency}

Power efficiency is used as the third schedule performance indicator and is related to the mean transmit 


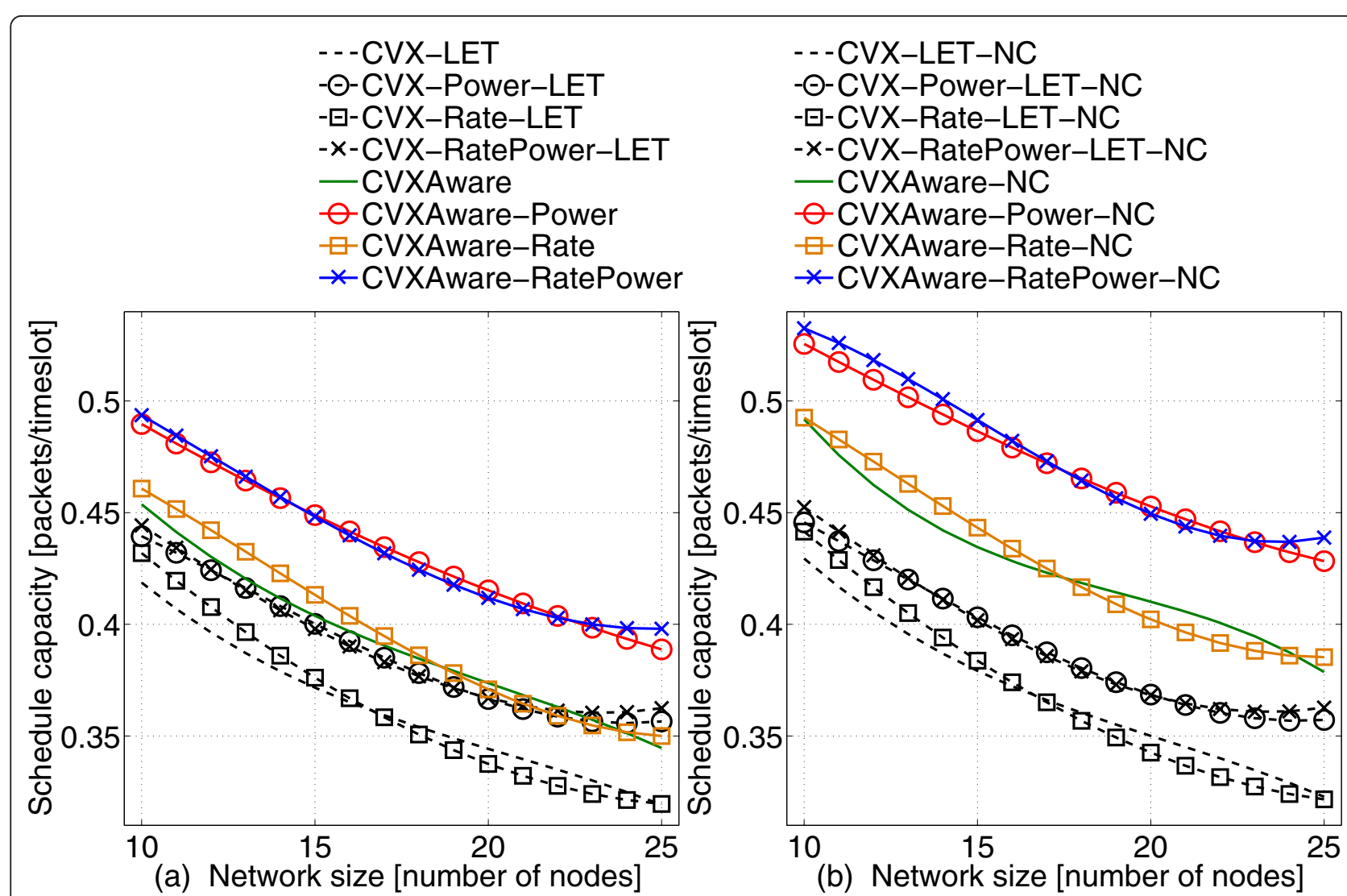

Figure 4 Schedule capacity comparison of power and rate adaptive schedules. (a) Scheduling without NC; (b) Scheduling with NC.

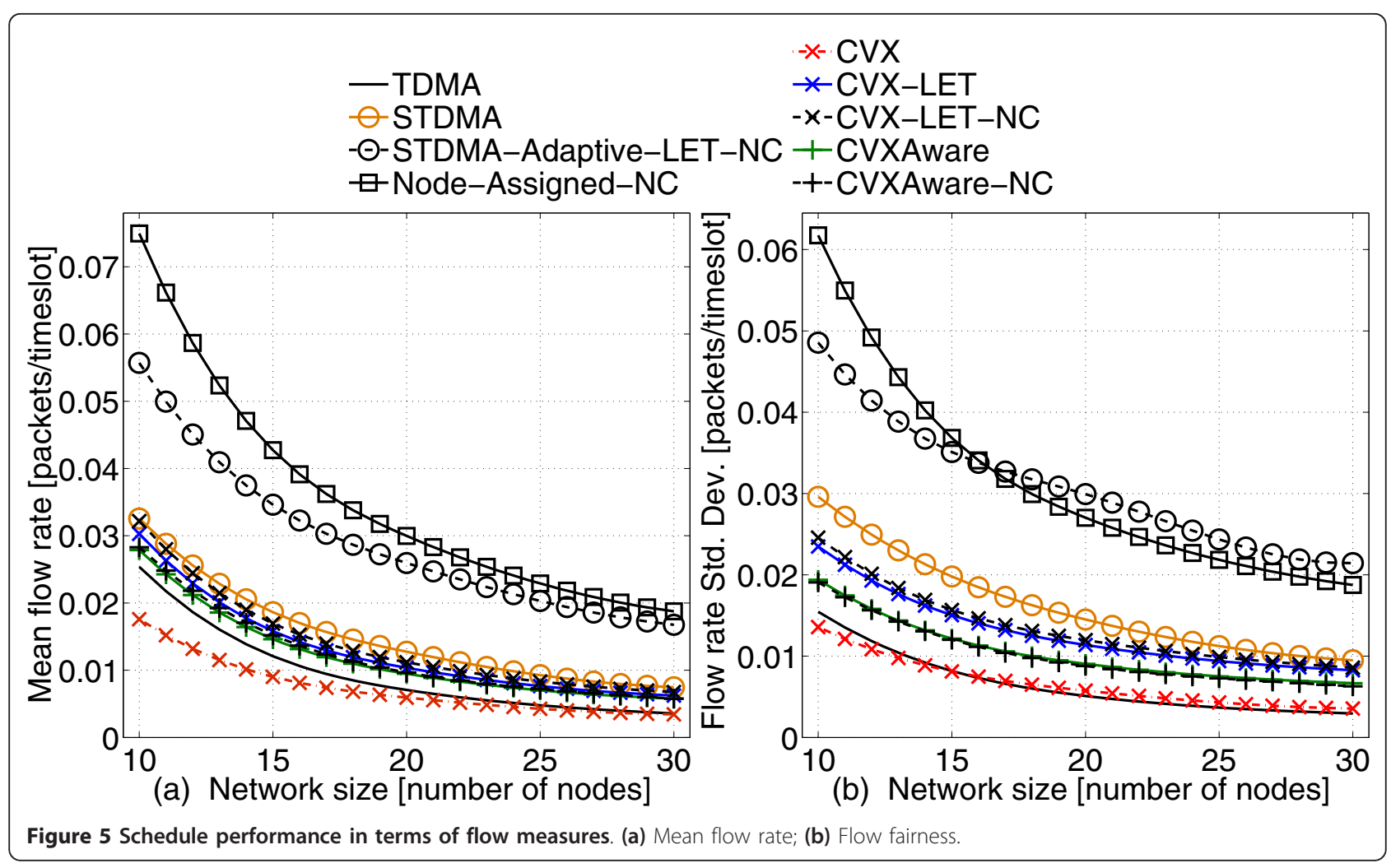




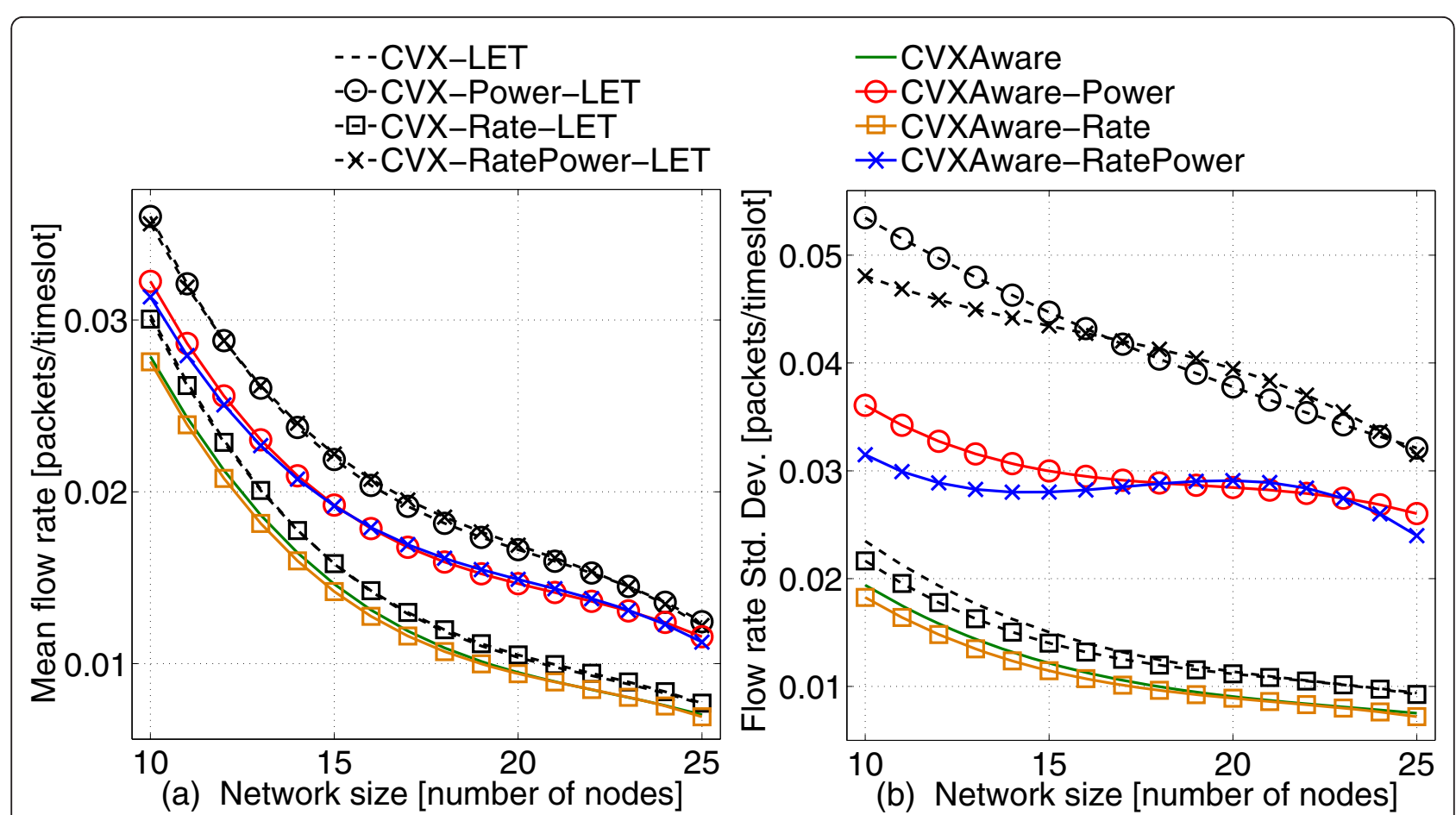

Figure 6 Flow measure comparison of schedules with variable power and rate control. (a) Mean flow rate; (b) Flow fairness.

power per packet. Adaptive power assignments, multirate transmissions and network coding can reduce the mean transmit power per packet of the full power case (CVX-LET) shown in Figure 7. By performing network coding (CVX-LET-NC), one transmission can relay two packets, so only half the energy is used in that case, which improves the power efficiency. Significantly less power is used with the power-adaptive schedules, and when network coding is used, the temporal reuse-aware schedules achieve greater added efficiency than the nonaware schedules.

\subsection{Spatial and temporal reuse}

There is a relationship between spatial and temporal reuse, since greater spatial reuse implies greater interference and consequently smaller temporal reuse. These results are evident in Figure 8, where the power-adaptive schedules have the greatest spatial reuse (Figure 8a) but the smallest temporal reuse (Figure $8 \mathrm{~b}$ ). Nodeassigned schedules in turn achieve the highest temporal reuse, which reduces the spatial reuse possibilities, so that these also achieve the smallest spatial reuse. The power-adaptive $\widetilde{\mathcal{C}}_{e}$-schedules give greater preference to temporal reuse when compared to the $\mathcal{C}$-schedules, which have higher spatial reuse. Thus, with temporal reuse-aware optimisation a more beneficial balance is found between spatial and temporal reuse, which results in favourable network performance.

\section{Conclusion}

The research question that has been addressed in this study is as follows: What generic transmission scheduling algorithm can produce predefined link-assigned schedules that maximise schedule capacity with proportionally fair end-to-end flow rates for stationary multihop mesh networks with single-path minimum hop routing, rate and power adaptivity, both spatial and temporal reuse and network coding?

The following contributions were made in answering this question:

1) The hybrid node-link assignment strategy of [6] is re-evaluated in terms of a new concept that is defined here, namely temporal reuse, which is recognised as the time-domain analogue of spatial reuse.

2) A more specific characterisation of the schedules that could induce packet depletion is given than in the previous study of [8]. It is then shown that optimal schedules can potentially benefit from higher usage efficiency afforded by temporal reuse.

3) A new achievable rate region is formulated that provides better estimates of the increased link capacities due to temporal reuse. The rate region was derived to be generic, meaning that only information on the network topology and data flows are needed to calculate its capacity without having to simulate the network in question. 

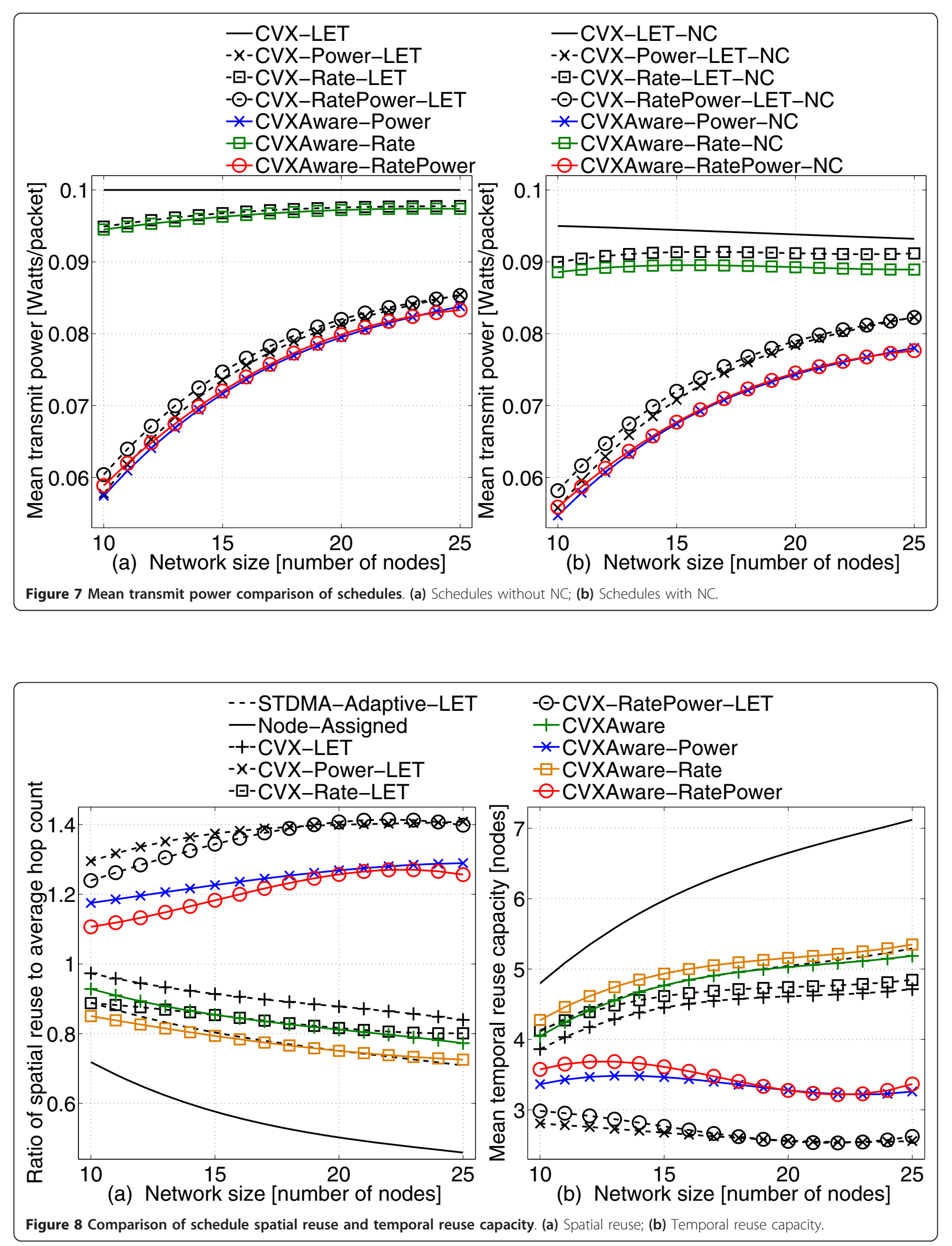
4) A cross-layer optimisation scheduling algorithm is given that uses the newly proposed rate region and solves the scheduling subproblem with temporal reuse in mind.

Firstly, it was experimentally shown that by optimising schedules with temporal reuse in the temporal reuseaware rate region, the schedule capacity is increased when compared to optimisation in the non-aware rate region of [9]. In addition, flow rate fairness is also improved with the proposed scheduler and the benefit derived from network coding is also greater. For schedules with rate and power adaptivity, it was shown that the proposed scheduler achieves better power efficiency.

The relationship between spatial and temporal reuse was analysed, and the temporal reuse-aware scheduler managed a more effective balance for the different rate and power adaptivities. The trade-off between schedule capacity and fairness was affirmed, and it was shown that the proposed class of optimised link-assigned schedules outperforms node-assigned schedules in that respect.

The research in this paper thus includes a high-performance scheduler for modern wireless mesh networks that use recent enhancements, such as network coding and temporal reuse. Possible extensions of this work might include an analysis for networks with multi-path routing, cooperative coding and more complex $n$-way $(n>2)$ network coding.

\footnotetext{
Acknowledgements

This research was supported by the SENTECH chair in Broadband Wireless Multimedia Communications at the University of Pretoria. The authors wish
} to thank the reviewers for their insightful suggestions and advice.

\section{Competing interests}

The authors declare that they have no competing interests.

Received: 22 November 2010 Accepted: 1 September 2011 Published: 1 September 2011

\section{References}

1. I Cidon, M Sidi, Distributed assignment algorithms for multihop packet radio network. IEEE Trans Comput. 38(4), 456-460 (1990). doi:10.1109/ 26.52656

2. I Chlamtac, S Kutten, A spatial reuse TDMA/FDMA for mobile multi-hop radio networks, in Proceedings of the IEEE InfoCom '85, 1, 389-394 (1985)

3. B Hajek, G Sasaki, Link scheduling in polynomial time. IEEE Trans Inform Theory, 35(5), 910-917 (1988)

4. I Chlamtac, A Lerner, A link allocation protocol for mobile multi-hop radio networks, in Proceedings of the IEEE GlobeCom '85, 1, 238-242 (1985)

5. L Pond, $V$ Li, A distributed time-slot assignment protocol for multi-hop broadcast packet radio networks. IEEE MilCom. 1, 70-74 (1989)

6. J Grönkvist, Novel assignment strategies for spatial reuse TDMA in wireless ad hoc networks. Wirel Netw. 12(2), 255-265 (2006). doi:10.1007/s11276005-5273-z

7. A Dhamdhere, J Grönkvist, Joint node and link assignment in an STDMA network, in IEEE 65th Vehicular Technology Conference 2007, 1066-1070 (2007)
8. FPS Luus, BT Maharaj, Cross-layer optimization of wireless networks with extended transmission rights, in Proceedings of the GlobeCom '10, (Miami, USA, December 2010)

9. M Johansson, L Xiao, Cross-layer optimization of wireless networks using nonlinear column generation. IEEE Trans Wirel Commun. 5(2), 435-445 (2006)

10. B Johansson, P Soldati, M Johansson, Mathematical decomposition techniques for distributed cross-layer optimization of data networks. IEEE J Sel Areas Commun. 24(8), 1535-1547 (2006)

11. P Gupta, PR Kumar, The capacity of wireless networks. IEEE Trans Inf Theory, 46, 388-404 (2000). doi:10.1109/18.825799

12. S Toumpis, AJ Goldsmith, Capacity regions for wireless ad hoc wireless networks. IEEE Trans Wirel Commun. 2(4), 736-748 (2003)

13. B Radunovic, J-YL Boudec, Joint scheduling, power control and routing in symmetric one-dimensional, multi-hop wireless networks, in Proceedings of WiOpt'03, (Sophia Antipolis, France, 2003), pp. 31-42

14. FP Kelly, AK Malulloo, DKH Tan, Rate control in communications networks: shadow prices, poportional fairness and stability. J Oper Res Soc. 49, 237-252 (1998)

15. C Carathéodory, Über den Variabilitätsbereich der Fourierschen Konstanten von positiven harmonischen Funktionen. Rend Circ Mat Palermo. 32, 193-217 (1911). doi:10.1007/BF03014795

16. B Radunovic, J-YL Boudec, Rate performance objectives of multihop wireless networks. IEEE Trans Mobile Comput. 3(4), 334-349 (2004). doi:10.1109/TMC.2004.45

17. P Värbrand, P Björklund, D Yuan, Resource optimization of spatial TDMA in ad hoc radio networks: a column generation approach, in Proceedings of IEEE InfoCom 2003, (San Francisco, March 2003)

18. SH Low, DE Lapsley, Optimization flow control-I:Basic algorithm and convergence. IEEE/ACM Trans Netw. 7(6), 861-874 (1999). doi:10.1109/ 90.811451

19. A Wächter, LT Biegler, On the implementation of a primal-dual interior point filter line search algorithm for large-scale nonlinear programming. Math Program. 106(1), 25-57 (2006). doi:10.1007/s10107-004-0559-y

20. S Katti, H Rahul, W Hu, D Katabi, M Medard, J Crowcroft, XORs in the air: practical network coding, in Proceedings of ACM SigComm 2006, 243-254 (2006)

21. K Papadaki, V Friderikos, Approximate dynamic programming for link scheduling in wireless mesh networks. Comput Oper Res. (2007)

22. J Grönkvist, Traffic controlled spatial reuse TDMA in multi-hop radio networks. PIMRC, 1203-1207 (1998)

doi:10.1186/1687-1499-2011-84

Cite this article as: Luus and Maharaj: Transmission scheduling for wireless mesh networks with temporal reuse. EURASIP Journal on Wireless Communications and Networking 2011 2011:84.

\section{Submit your manuscript to a SpringerOpen ${ }^{\circ}$ journal and benefit from:}

- Convenient online submission

- Rigorous peer review

- Immediate publication on acceptance

- Open access: articles freely available online

- High visibility within the field

- Retaining the copyright to your article

Submit your next manuscript at $\boldsymbol{s p r i n g e r o p e n . c o m ~}$ 